\title{
Tumor-associated macrophages predict prognosis in diffuse large B-cell lymphoma and correlation with peripheral absolute monocyte count
}

\author{
Yan-Li Li ${ }^{1,2}$ D, Zhi-Hu Shi ${ }^{3}$, Xian Wang ${ }^{1,4}$, Kang-Sheng Gu ${ }^{5}$ and Zhi-Min Zhai ${ }^{6^{*}}$
}

\begin{abstract}
Background: Diffuse large B-cell lymphoma (DLBCL) is characterized by its clinical and biological heterogeneity. The clinical prognostic implications of tumor-associated macrophages (TAMs) in DLBCL remain controversial and the correlation between TAMs and peripheral absolute monocyte count (AMC) has not yet been elucidated.

Methods: In 221 untreated, newly diagnosed patients with DLBCL, we evaluated the prognostic value of TAMs using immunohistochemical analysis, as well as the association of TAMs and AMC.

Results: We found that high CD68 or high CD163 expression was correlated with clinicopathological characteristics, high CD163 expression was an adverse predictor for both overall survival (OS) [hazard ratio (HR) $=2.265, P=0.005$ ] and progression- free survival (PFS) $(H R=1.925, P=0.017)$ in patients with $D L B C L$. Patients with high CD68 or high CD163 expression had significantly poorer OS and PFS than those with low CD68 or low CD163 expression, respectively (CD68: OS: $P<0.001$, PFS: $P<0.001$; CD163: OS: $P<0.001$, PFS: $P<0.001$ ), even in the rituximab era. Moreover, high-risk patients could be further identified by the expression of CD68 or CD163, especially in those classified as low/intermediate risk by International Prognostic Index (IPI). Furthermore, the significant positive correlation was also detected between CD68 expression or CD163 expression and AMC $(r=0.256, P<0.001 ; r=0.303$, $P<0.001)$.

Conclusions: Patients with high expression of TAMs tend to have poorer OS and PFS, even in the rituximab era, and have positive correlation with AMC. Therefore, the peripheral AMC is a useful prognostic marker reflecting the status of the tumor microenvironment (TME) in DLBCL.
\end{abstract}

Keywords: Diffuse large B-cell lymphoma, Tumor-associated macrophages, Absolute monocyte count, Prognosis

\section{Background}

Diffuse large B-cell lymphoma (DLBCL) is the most common adult non-Hodgkin lymphoma (NHL), accounting for $30-40 \%$. Although aggressive, it can be cured in $60-70 \%$ of cases after first-line immunochemotherapy. Nevertheless, $30-40 \%$ of cases will experience recurrence or refractory disease after initial response, which will dramatically reduce their survival time. These patients remain a challenging therapeutic problem, and

\footnotetext{
*Correspondence: zzzm889@163.com

${ }^{6}$ Department of Hematology, The Second Affiliated Hospital of Anhui Medical University, Hefei, Anhui 230601, People's Republic of China Full list of author information is available at the end of the article
}

more targeted and personalized approach is an important goal for them, which is still being explored [1].

In the past decades, the view that tumors mainly consist of tumor cells has changed, and tumors are now thought to have organoid structures. In addition to tumor cells, there are many other tumor-infiltrating stromal cells, such as fibroblasts, immune cells, vascular endothelial cells and mesenchymal stem cells, which interact closely with tumor cells, constituting the tumor microenvironment (TME) [2]. TME influences initiation, growth and metastasis of tumors, including DLBCL.

Tumor-associated macrophages (TAMs), as the intrinsic cellular components of TME, involve in tumor

(c) The Author(s). 2019 Open Access This article is distributed under the terms of the Creative Commons Attribution 4.0 International License (http://creativecommons.org/licenses/by/4.0/), which permits unrestricted use, distribution, and 
proliferation, invasion, angiogenesis, metastasis and suppression of anti-tumor immunity in many tumors [3]. In general, macrophages (MPs) often have two kinds of phenotypes because of different activation states. One is "classically" activated M1 phenotype with antitumor activity, the other is "alternatively" activated M2 phenotype with tumor-promoting activity [4]. So far, the clinical prognostic significance of TAMs in DLBCL remains controversial mainly due to difference in the method used to evaluate MPs as well as types of treatment given [5-13].

Another known prognostic marker in DLBCL is the peripheral absolute monocyte count (AMC), and elevated AMC levels have been reported to correlate with poor prognosis in DLBCL patients [14-20]. It is widely known that the majority of TAMs are derived from peripheral monocytes, however, the correlation between TAMs and AMC has not yet been elucidated in DLBCL. Therefore, in this study, we evaluated the predictive value of TAMs and clarified the correlation between TAMs and AMC in DLBCL patients.

\section{Methods}

\section{Ethics statement}

This study was carried out in accordance with the principles of the Helsinki Declaration and approved by the ethics committee of the first affiliated and the second affiliated hospitals of Anhui Medical University. Each patient had signed informed consent.

\section{Patients}

two hundred twenty-one untreated, newly diagnosed patients with DLBCL were recruited between 2004 and 2015 from the First Affiliated Hospital and the Second Affiliated Hospital of Anhui Medical University, excluding immunodeficiency, transformed DLBCL, primary central nervous system DLBCL, EBV+ DLBCL, HHV8+ DLBCL and primary cutaneous DLBCL, leg type. For diagnosis and prognostic purposes, the current World Health Organization classification [21] and International Prognostic Index (IPI) [22] were used. All patients were treated with CHOP (cyclophosphamide, hydroxydaunorubicin, vincristine, prednisone) or R-CHOP (rituximabcyclophosphamIde, hydroxydaunorubicin, vincristine, prednisone) every 3 weeks for 3 to 8 cycles as first-line therapy, and follow-up in the hospitals.

\section{Blood sample analysis}

The AMC at diagnosis was obtained from routine automated complete blood count $(\mathrm{CBC})$, and the cut-point for the AMC at diagnosis was $460 / \mu \mathrm{l}$ based on our previous data [14].

\section{Immunohistochemistry}

two hundred twenty-one specimens for immunohistochemical analysis were formalin-fixed and paraffin- embedded. 4-um-thick paraffin sections and monoclonal antibodies against human CD68 (1:400 dilution, KP1, OriGene, USA) and CD163 (1300 dilution, 10D6, OriGene, USA) were used in the study. The specimen sections were deparaffinized in xylene and rehydrated in a series of grade alcohols. They were then protreated in citric acid antigen retrieval solution ( $\mathrm{pH}$ 6.0) using heatinduced epitope retrieval technique. After inhibiting internal peroxidase activity with $3 \%$ hydrogen peroxide, the sections were incubated with anti-CD68 antibody and anti-CD163 antibody overnight at $4{ }^{\circ} \mathrm{C}$. The slides were then incubated with HRP-conjugated goat antimouse IgG secondary antibody for $10 \mathrm{~min}$ at $37^{\circ} \mathrm{C}$. Finally, the sections were visualised by $\mathrm{DAB}$ solution (DAKO, Carpinteria, CA, USA) and counterstained with haematoxylin (DAKO). CD68 positive staining was observed in cytoplasm of MPs, and CD163 positive staining was observed in membrane of MPs. Two professional pathologists who did not know the clinical data analysed all stained sections by quantitative method. The average count of five high-power fields $(\mathrm{HPF})(\times 400$, an area of view $0.24 \mathrm{~mm}^{2}$ ) for each slice was obtained by an Olympus BX51TF microscope (Olympus, Japan).

\section{Statistical analysis}

Receiver operating characteristics (ROC) analysis and area under the curve (AUC) were used to determine the optimal cut-point for the TAMs $[9,12]$. The correlation between TAMs and clinical characteristics or AMC used the chi-square test or Fisher's exact test. Univariate and multivariate analyses using Cox proportional hazards model were performed to explore whether TAMs was prognostic factors for survival when in combination with other clinicopathological factors. Overall survival (OS) was defined as the time from diagnosis until death or the last follow-up.Progression-free survival (PFS) was defined as the time from diagnosis until disease progression, relapse, death of any cause or the last follow-up. OS and PFS were estimated using the Kaplan-Meier method and compared using the log-rank test. $P$ values< 0.05 were determined to be statistically significant. Statistical analysis was performed using SPSS 17.0 software (SPSS Inc., Chicago, IL, USA).

\section{Results}

Immunohistochemical CD68 and CD163 intensity and cutpoints for CD68 + cells and CD163 + cells

By immunohistochemical analysis, in tumor tissue, the median level of CD68 + cells/ HPF was 27 (range, 7-83), and the median level of CD163 + cells/HPF was 17 (range, $2-78$ ). Based on survival information (death/survival at 5 
years after diagnosis), the ROC curves and AUC were used to determine their cut-points. The most optimal cut-point of CD68 + cells was 33/HPF, with an AUC value of 0.706 (95\%CI, 0.638-0.774, $P<0.001$ ) (Additional file 1: Figure S1A); the most optimal cut-point of CD163+ cells was 19/ HPF, with an AUC value of 0.729 (95\%CI, 0.663-0.795, $P<0.001$ ) (Additional file 1: Figure S1B). Of 221 DLBCL patients, $138(62.4 \%)$ were categorized as low CD68 expression $(\mathrm{CD} 68+$ cells $<33 / \mathrm{HPF})$ and $83(37.6 \%)$ as high CD68 expression (CD68 + cells $\geq 33 / \mathrm{HPF}$ ) (Fig. 1a, b), 121 (54.8\%) were categorized as low CD163 expression $(\mathrm{CD} 163+$ cells $<19 / \mathrm{HPF})$ and $100(45.2 \%)$ as high CD163 expression (CD163 + cells $\geq 19 /$ HPF) (Fig. 1c, d).

The clinicopathological characteristics according to CD68 or CD163 expression and correlation of CD68 or CD163 expression with AMC

The median age of 221 DLBCL patients was 56 years (range, 13-84 years). The median follow-up time after diagnosis was 42 months (range, 3-118 months). The clinico- pathological characteristics are showed in Table 1. High CD68 expression was significantly correlated with poor ECOG-PS $(P<0.001)$, more extranodal sites of disease $(P=0.014)$, III/IV Ann Arbor stage $(P<0.001)$, elevated LDH $(P<0.001), 3-5$ IPI score $(P<0.001)$ and high AMC $(P<0.001)$, and high CD163 expression was significantly correlated with poor ECOG-PS $(P=0.018)$, more extranodal sites of disease $(P=0.026)$, III/IV Ann Arbor stage
$(P=0.004)$, elevated LDH $(P<0.001), 3-5$ IPI score $(P<$ $0.001)$ and high AMC $(P<0.001)$ (Table 1$)$. The frequencies of CD68 and CD163 expression level significantly increased gradually accompanied with IPI score (CD68: $P<0.001$, Additional file 1: Figure S2A; CD163: $P<0.001$, Additional file 1: Figure S2B).

Significant positive associations were found between CD68 expression and CD163 expression $(r=0.766, P<$ 0.001, Fig. 2a), between CD68 expression and AMC $(r=$ 0.256, $P<0.001$, Fig. 2b), CD163 expression and AMC $(r=0.303, P<0.001$, Fig. 2c) by spearman correlation analysis.

\section{High CD163 expression is an independent adverse predictor for survival}

Univariate and multivariate analyses were performed to explore whether CD68 or CD163 expression was an independent prognostic factor for survival when in combination with other clinicopathological factors. Old age, poor ECOG-PS, more extranodal sites of disease, III/IV Ann Arbor stage, elevated LDH, 3-5 IPI score, high AMC, without rituximab therapy, high CD68 expression and high CD163 expression were all significant prognostic factors for poor survival by univariate analyses. The above-mentioned significant clinicopathological factors were entered into multivariate analysis (Table 2). As with III/IV Ann Arbor stage (OS: $P=0.047$, PFS: $P=$ 0.010), elevated LDH (OS: $P=0.005$, PFS: $P=0.007$ ),
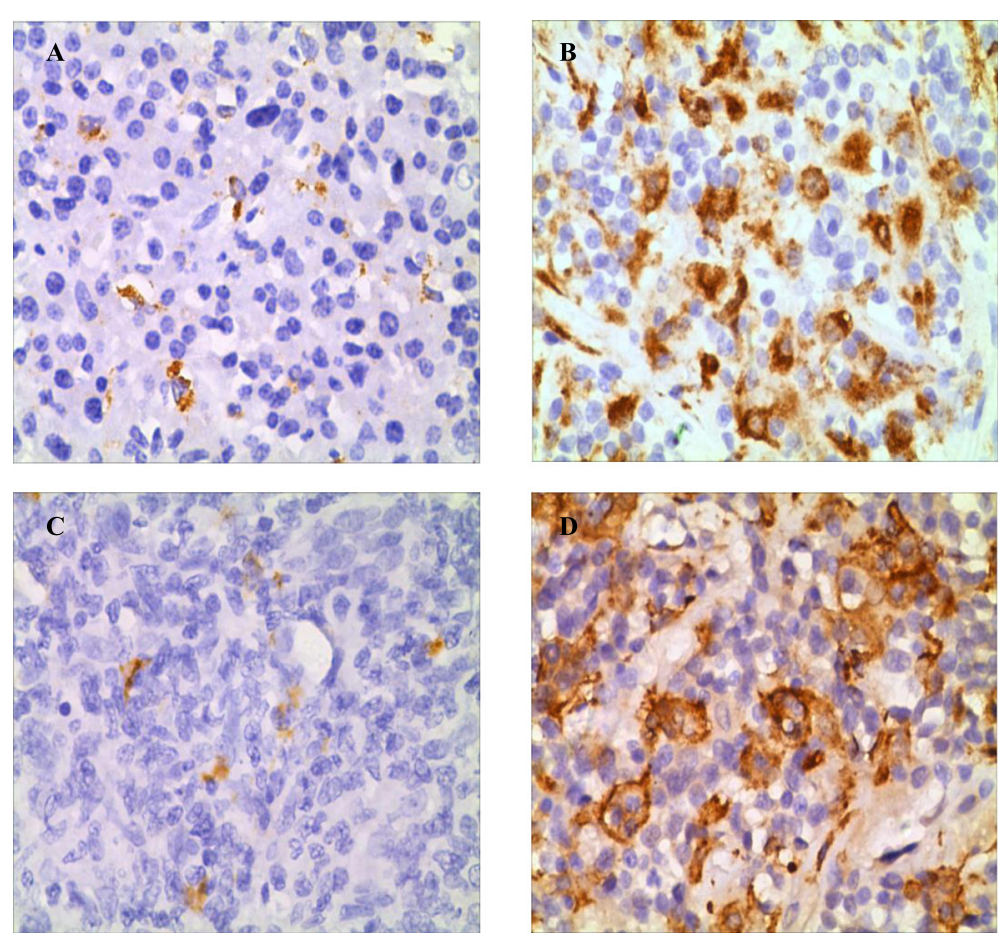

Fig. 1 CD68 and CD163 expression in DLBCL tissues (×400). a low CD68 expression, b high CD68 expression, c low CD163 expression, d high CD163 expression 
Table 1 Patient's demographics according to CD68 and CD163 expression

\begin{tabular}{|c|c|c|c|c|c|c|c|c|}
\hline \multirow[t]{2}{*}{ Characteristics } & \multicolumn{2}{|c|}{ Patients } & \multicolumn{3}{|c|}{ CD68 expression } & \multicolumn{3}{|c|}{ CD163 expression } \\
\hline & $\mathrm{NO}$. & $\%$ & Low & High & $P$ & Low & High & $P$ \\
\hline \multicolumn{9}{|l|}{ Gender } \\
\hline Female & 105 & 47.5 & 70 & 35 & 0.217 & 62 & 43 & 0.222 \\
\hline Male & 116 & 52.5 & 68 & 48 & & 59 & 57 & \\
\hline \multicolumn{9}{|l|}{ Age (years) } \\
\hline$\leq 60$ & 130 & 58.8 & 87 & 43 & 0.100 & 73 & 57 & 0.617 \\
\hline$>60$ & 91 & 41.2 & 51 & 40 & & 48 & 43 & \\
\hline \multicolumn{9}{|l|}{ ECOG-PS } \\
\hline$\leq 1$ & 136 & 61.5 & 98 & 38 & $<0.001$ & 83 & 53 & 0.018 \\
\hline$>1$ & 85 & 38.5 & 40 & 45 & & 38 & 47 & \\
\hline \multicolumn{9}{|c|}{ Extranodal sites of disease } \\
\hline$\leq 1$ & 176 & 79.6 & 117 & 59 & 0.014 & 103 & 73 & 0.026 \\
\hline$>1$ & 45 & 20.4 & 21 & 24 & & 18 & 27 & \\
\hline \multicolumn{9}{|c|}{ Ann Arbor stage } \\
\hline$|/| \mid$ & 127 & 57.5 & 93 & 34 & $<0.001$ & 80 & 47 & 0.004 \\
\hline III/IV & 94 & 42.5 & 45 & 49 & & 41 & 53 & \\
\hline \multicolumn{9}{|l|}{ LDH } \\
\hline$\leq$ normal & 159 & 71.9 & 114 & 45 & $<0.001$ & 103 & 56 & $<0.001$ \\
\hline >normal & 62 & 28.1 & 24 & 38 & & 18 & 44 & \\
\hline \multicolumn{9}{|l|}{ IPI score } \\
\hline $0-2$ & 154 & 69.7 & 114 & 40 & $<0.001$ & 98 & 56 & $<0.001$ \\
\hline $3-5$ & 67 & 30.3 & 24 & 43 & & 23 & 44 & \\
\hline \multicolumn{9}{|l|}{ AMC } \\
\hline$<460 /$ ul & 118 & 53.4 & 88 & 30 & $<0.001$ & 82 & 36 & $<0.001$ \\
\hline$\geq 460 / u l$ & 103 & 46.6 & 50 & 53 & & 39 & 64 & \\
\hline
\end{tabular}

Abbreviations: ECOG-PS Eastern Cooperative Oncology Group performance status, LDH lactate dehydrogenase, IPI International Prognostic Index, AMC absolute monocyte count high AMC (OS: $P=0.011$, PFS: $P=0.008$ ), and without rituximab therapy (OS: $P=0.003$, PFS: $P=0.010$ ), we found that high CD163 expression was an independent adverse predictor for both OS and PFS (OS: $P=0.005$, PFS: $P=0.017)$.

\section{Prognostic value of CD68 expression and CD163 expression in DLBCL patients}

In comparison with low expression of CD68, shorter OS and PFS could be found in DLBCL patients with high expression of CD68 (median OS: 19 vs 41 months, $P<$ 0.001; median PFS: 11 vs 27 months, $P<0.001$ ). Meanwhile, DLBCL patients with high expression of CD163 had significantly poorer OS and PFS than those with low expression of CD163 (median OS: 19 vs 44 months, $P<$ 0.001; median PFS: 13 vs 28 months, $P<0.001$ ). We further examined whether CD68 or CD163 expression could identify high-risk patients in different IPI score subgroups including low risk (score $=0-1$ ), intermediate risk (score $=2-3$ ) and high risk (score $=4-5)$. In low risk group $(n=113)$, high-risk patients could be significantly identified by CD68 expression (median OS: 25 vs 46 months, $P=0.002$, Fig. 3a; median PFS: 16 vs 32 months, $P=0.001$, Fig. 3 d) or CD163 expression (median OS: 24 vs 50 months, $P<0.001$, Fig. 4a; median PFS: 17 vs 34 months, $P<0.001$, Fig. $4 \mathrm{~d})$. The intermediate risk $(n=77)$ patients were equally identified using CD68 expression (median OS: 17 vs 36 months, $P<0.001$, Fig. 3 b; median PFS: 10 vs 22 months, $P<0.001$, Fig. $3 e$ ) or CD163 expression (median OS: 17 vs 37 months, $P<0.001$, Fig. $4 \mathrm{~b}$; median PFS: 10 vs 23 months, $P<0.001$, Fig. 4e). However, in high risk group $(n=31)$, CD68 or CD163 expression was not significantly predictive in the study (CD68: median OS: 13 vs 20 months, $P=0.573$, Fig. 3c; median

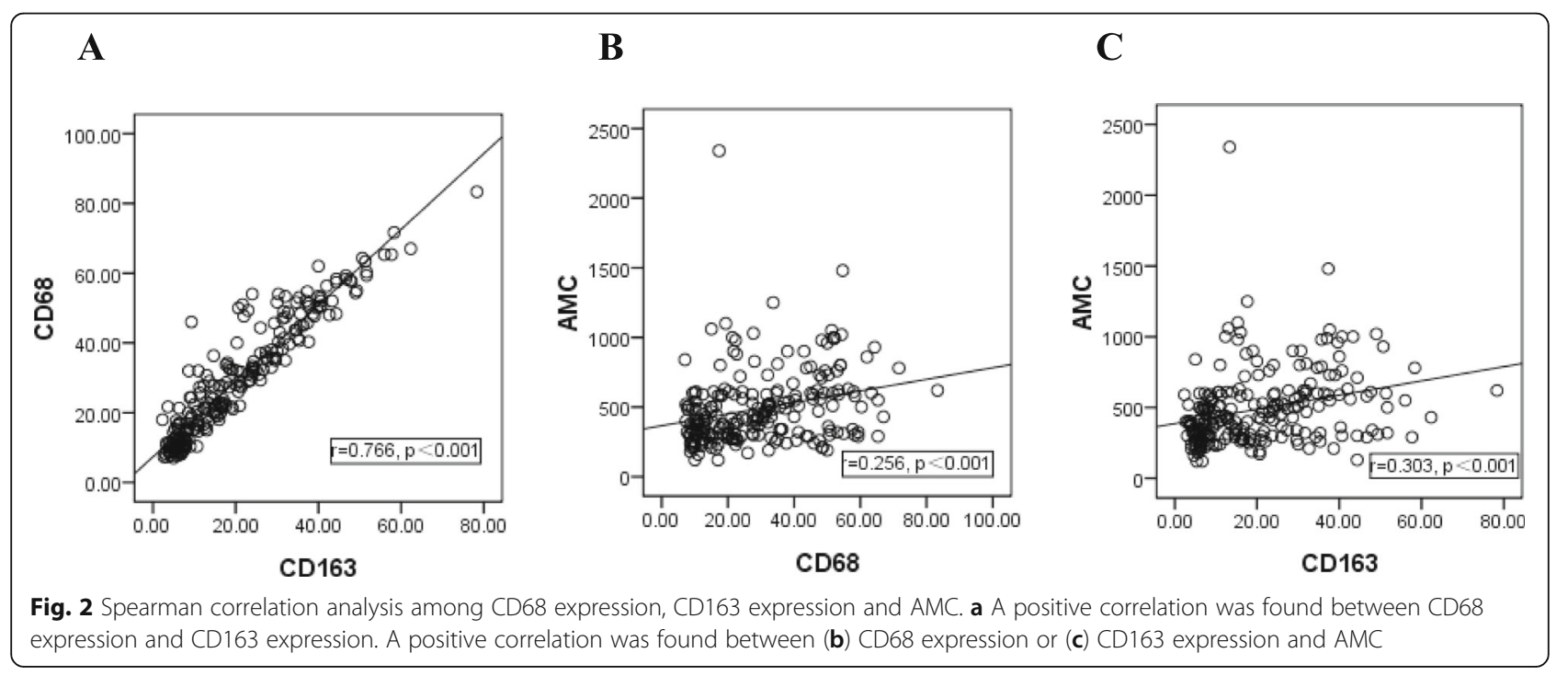


Table 2 Multivariate Cox regression analyses of potential prognostic factors for OS and PFS

\begin{tabular}{|c|c|c|c|c|}
\hline \multirow[t]{2}{*}{ Variables } & \multicolumn{2}{|l|}{ OS } & \multicolumn{2}{|l|}{ PFS } \\
\hline & $\mathrm{HR}(95 \% \mathrm{Cl})$ & $P$ & $\mathrm{HR}(95 \% \mathrm{Cl})$ & $P$ \\
\hline \multicolumn{5}{|l|}{ Age } \\
\hline $\begin{array}{l}\leq 60 \text { years/ } \\
>60 \text { years }\end{array}$ & $0.987(0.674-1.444)$ & 0.946 & $0.835(0.562-1.241)$ & 0.373 \\
\hline \multicolumn{5}{|l|}{ ECOG-PS } \\
\hline $1 />1$ & $1.126(0.725-1.749)$ & 0.596 & $1.037(0.668-1.610)$ & 0.872 \\
\hline \multicolumn{5}{|l|}{ Extranodal sites } \\
\hline$\leq 1 />1$ & $1.019(0.610-1.705)$ & 0.941 & $1.038(0.621-1.733)$ & 0.888 \\
\hline \multicolumn{5}{|c|}{ Ann Arbor stage } \\
\hline lorll/IIIorlV & $1.620(1.006-2.606)$ & 0.047 & $1.862(1.157-2.995)$ & 0.010 \\
\hline \multicolumn{5}{|l|}{ LDH } \\
\hline $\begin{array}{l}\leq \text { normal/ } \\
>\text { normal }\end{array}$ & $1.881(1.214-2.916)$ & 0.005 & $1.815(1.173-2.810)$ & 0.007 \\
\hline \multicolumn{5}{|l|}{ IPI score } \\
\hline $0-2 / 3-5$ & $1.167(0.562-2.424)$ & 0.679 & $1.320(0.631-2.760)$ & 0.461 \\
\hline \multicolumn{5}{|l|}{ AMC } \\
\hline $\begin{array}{l}<460 / \text { ul/ } \\
\geq 460 / \text { ul }\end{array}$ & $1.658(1.124-2.446)$ & 0.011 & $1.675(1.142-2.457)$ & 0.008 \\
\hline \multicolumn{5}{|l|}{ Treatment } \\
\hline $\begin{array}{l}\text { R-CHOP/ } \\
\text { CHOP }\end{array}$ & $1.978(1.269-3.082)$ & 0.003 & $1.780(1.149-2.756)$ & 0.010 \\
\hline \multicolumn{5}{|l|}{ CD68 } \\
\hline low/high & $1.237(0.710-2.156)$ & 0.453 & 1.369 (0.789-2.376) & 0.264 \\
\hline \multicolumn{5}{|l|}{ CD163 } \\
\hline low/high & $2.265(1.280-4.008)$ & 0.005 & $1.925(1.125-3.291)$ & 0.017 \\
\hline
\end{tabular}

Abbreviations: OS overall survival, PFS progression-free survival, $H R$ hazard ratio, Cl confidence interval, ECOG-PS Eastern Cooperative Oncology Group performance status, $L D H$ lactate dehydrogenase, IPI International Prognostic Index, $A M C$ absolute monocyte count, $C H O P$ cyclophosphamide hydroxydaunorubicin vincristine prednisone, $R$-CHOP rituximabcyclophosphamidehydroxydaunorubicin vincristine prednisone

PFS: 8 vs 11 months, $P=0.680$, Fig. 3f; CD163: median OS: 14 vs 19 months, $P=0.749$, Fig. 4c; median PFS: 8 vs 10 months, $P=0.823$, Fig. $4 \mathrm{f})$.

In addition, in DLBCL patients who received $\mathrm{R}-\mathrm{CHOP}$ $(n=59)$, high expression of CD68 or CD163 had significantly poorer OS and PFS than those with low expression of CD68 or CD163 (CD68: median OS: 23 vs 50 months, $P<0.001$; median PFS: 12 vs 33 months, $P<$ 0.001; CD163: median OS: 23 vs 54 months, $P<0.001$; median PFS: 14 vs 36 months, $P<0.001)$. In intermediate risk group $(n=19)$, high-risk patients could be identified by CD68 or CD163 expression (CD68: median OS: 18 vs 54 months, $P<0.001$, Fig. $5 \mathrm{a}$; median PFS: 8 vs 30 months, $P<0.001$, Fig. 5b; CD163: median OS: 19 vs 56 months, $P=0.002$, Fig. $5 \mathrm{c}$; median PFS: 8 vs 32 months, $P<0.001$, Fig. $5 d)$.

\section{Discussion}

The present study evaluated the clinical prognostic implications of TAMs in DLBCL, as well as the association with AMC. We used 2 markers to identify TAMs, CD68 and CD163, and found that high CD68 or CD163 expression was correlated with clinico- pathological characteristics, high CD163 expression was an adverse predictor for both OS and PFS. Patients with high CD68 or high CD163 expression had significantly poorer OS and PFS than those with low CD68 or low CD163 expression, respectively, even in the rituximab era. Moreover, high-risk patients could be further identified by the expression of CD68 or CD163, especially in those classified as low/intermediate risk by IPI. Furthermore, the significant positive correlation was also detected between TAMs and AMC.

A recognized hallmark of cancer is the non-resolving inflammation in TME. In addition to tumor cells, there are different stromal cells influencing the tumorigenesis, growth, metastasis and angiogenesis of tumors in TME, such as fibroblasts, leukocytes (including myeloid lineage cells and lymphoid lineage cells), vascular endothelial cells and mesenchymal stem cells. TAMs, generally thought to be more similar to M2- polarized MPs, are often prominent stromal cells that coordinate various factors in TME, and are also known as alternatively activated MPs, which are activated by helper T cell 2 (Th2) cytokines, such as interleukin (IL)-4, IL-10 and IL-13 [23]. In recent studies, TAMs in TME were found to correlate with clinical prognosis in hematologic neoplasms, particularly in Hodgkin's lymphoma (HL) [24]. Nevertheless, its significance in DLBCL has thus far been controversial. The number of CD68 + cells did not exhibit significant prognostic value [13], and was found to have no significant relation with OS or PFS [5], whereas other researches showed that higher CD68 + cells tended to predict poorer prognosis $[6,8,9]$ and worse treatment response [6, 11]. But recently, Nam et al. [8] and Riihijärvi S et al. [9] suggested that higher CD68 expression was correlated with better prognosis in DLBCL received rituximab in addition to other chemotherapy such as $\mathrm{CHOP}$, and with poorer outcome when rituximab was not given. Our study results are in line with these findings $[7,8]$, suggesting that high CD68 expression was correlated with clinicopathological characteristics and had significantly poor OS and PFS, even in the rituximab era. In addition, it is generally believed that high CD163 expression predicts poor prognosis in DLBCL patients $[7,8,10,12,13]$, and our results are consistent with those previous studies. The clinical prognostic significance of TAMs in DLBCL remains controversial possibly due to difference in clinical case-study method, patient populations, instrument (such as field of view) and method used to evaluate MPs as well as types of 
A

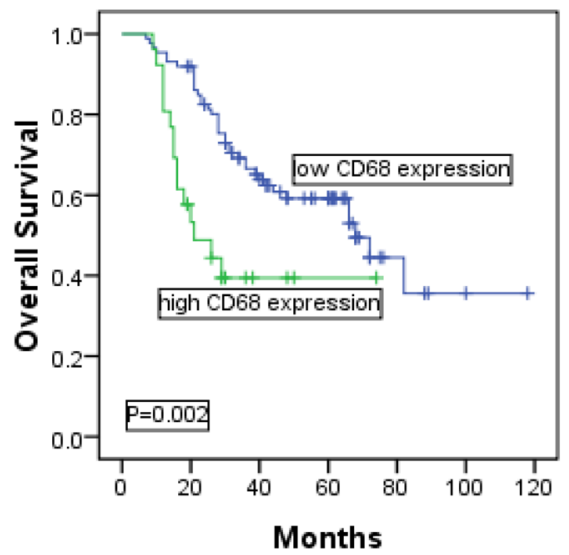

C

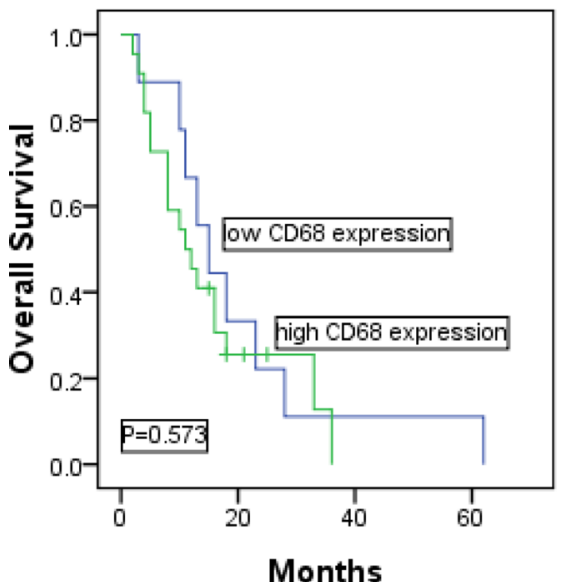

E

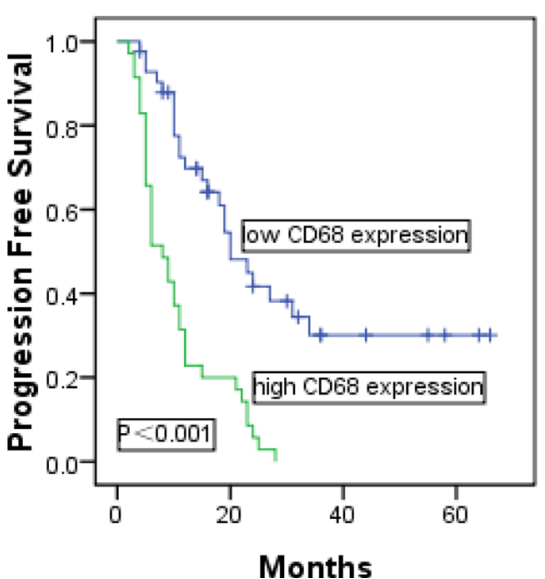

B

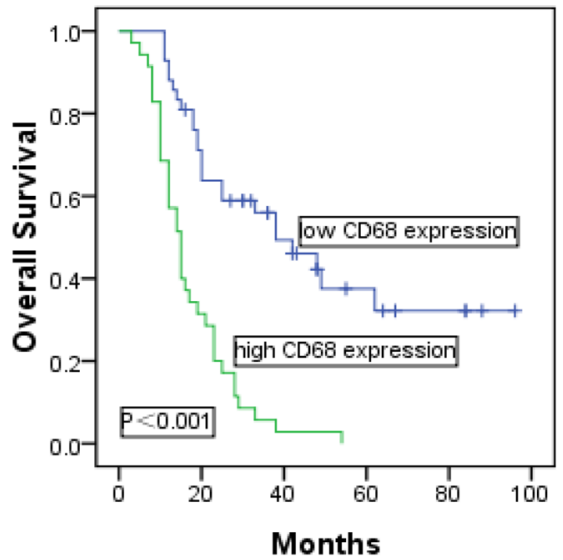

D

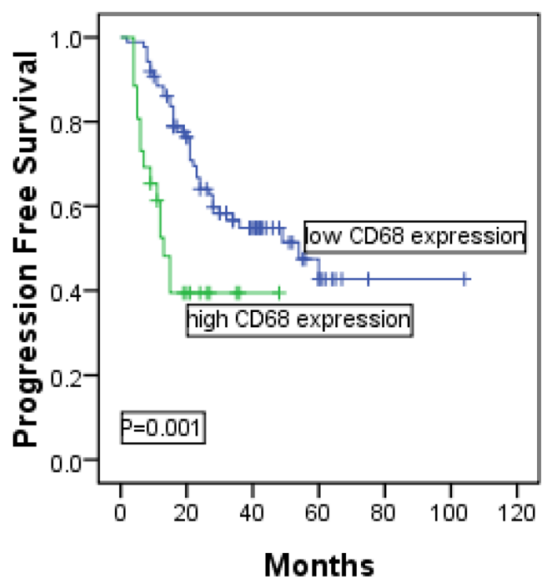

F

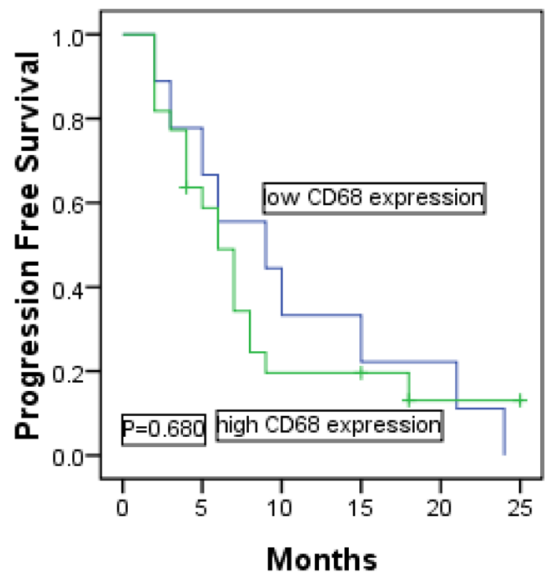

Fig. 3 Kaplan-Meier analysis of OS and PFS according to the expression of CD68 in patients with DLBCL. Patients identified by the IPI score as $(\mathbf{a}, \mathbf{d})$ IPI 0-1, (b,e) IPI 2-3, (c,f) IPI 4-5 were further stratified into low CD68 expression or high CD68 expression groups, respectively 
A

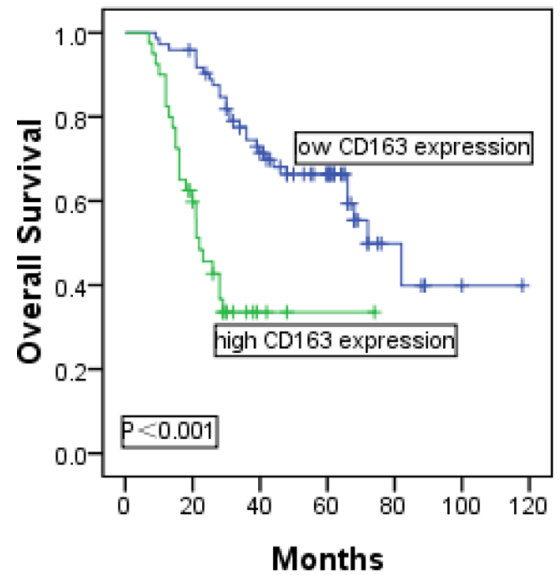

C

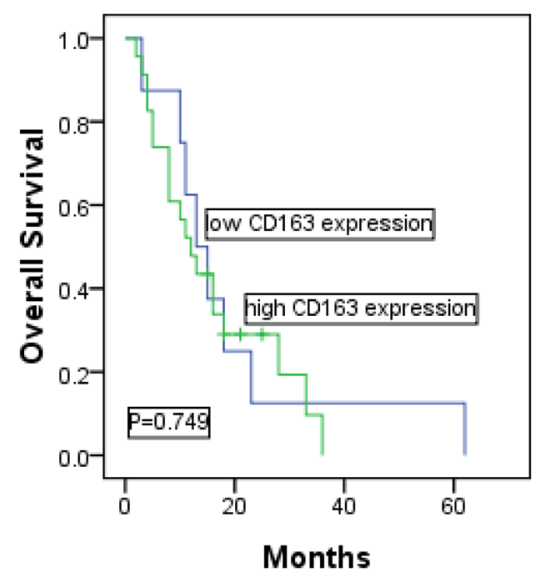

$\mathbf{E}$

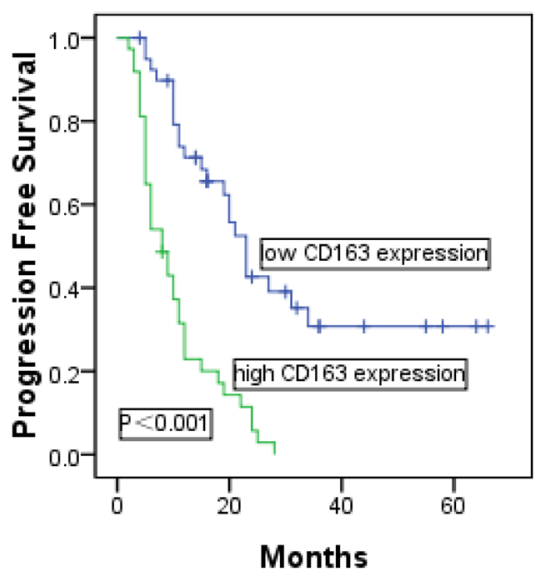

B

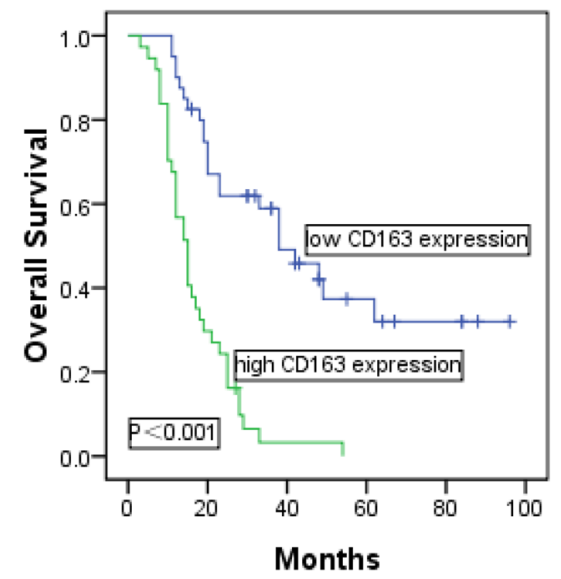

$\mathbf{D}$

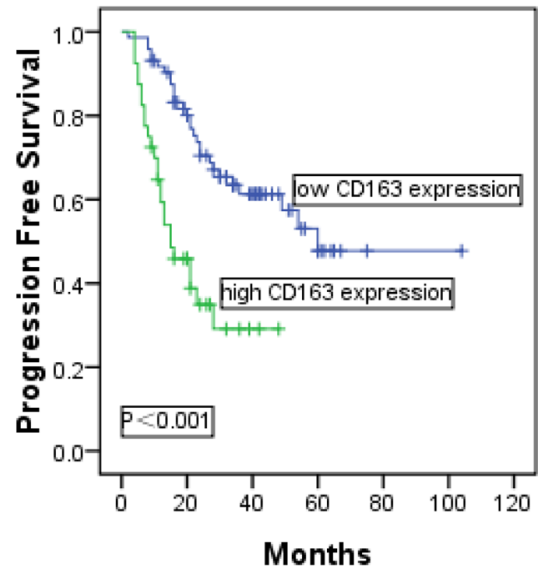

F

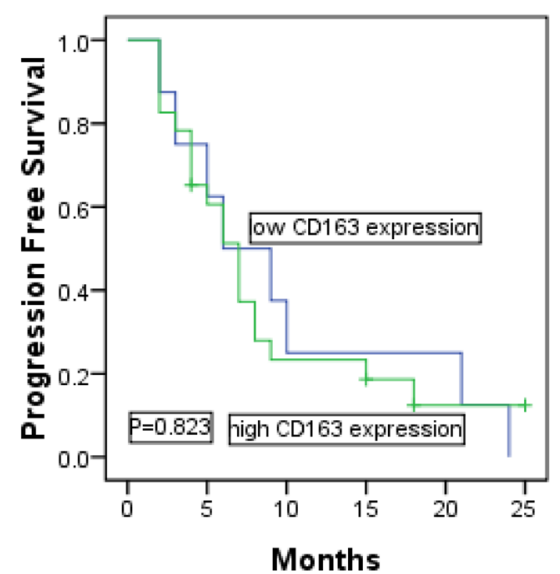

Fig. 4 Kaplan-Meier analysis of OS and PFS according to the expression of CD163 in patients with DLBCL. Patients identified by the IPI score as $(\mathbf{a}, \mathbf{d})$ IPI 0-1, (b,e) IPI 2-3, (c,f) IPI 4-5 were further stratified into low CD163 expression or high CD163 expression groups, respectively 
A

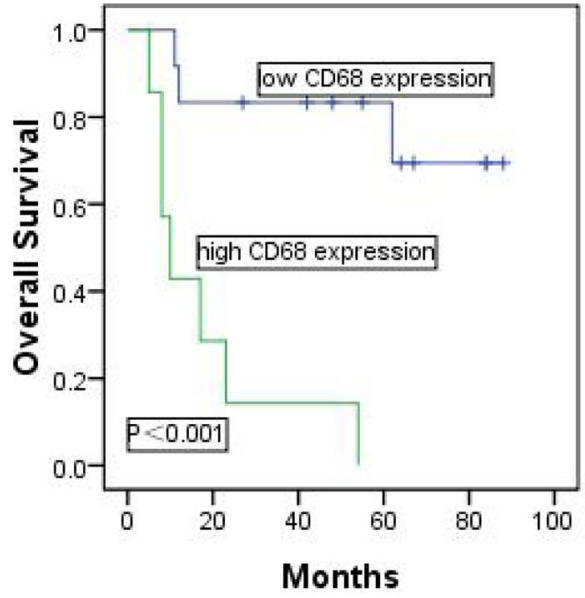

C

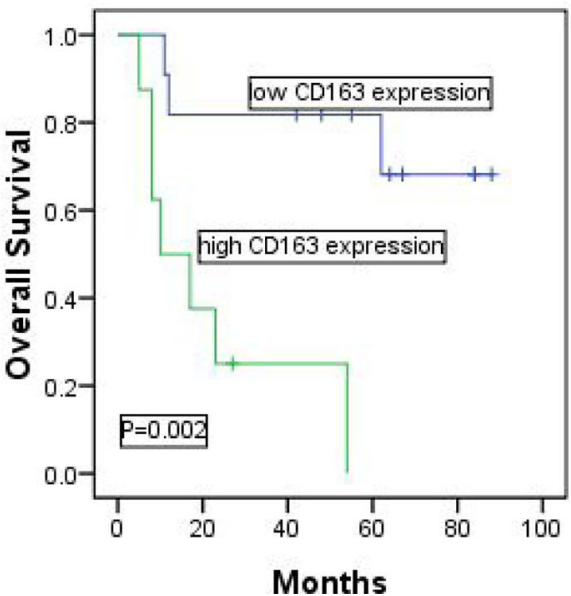

B

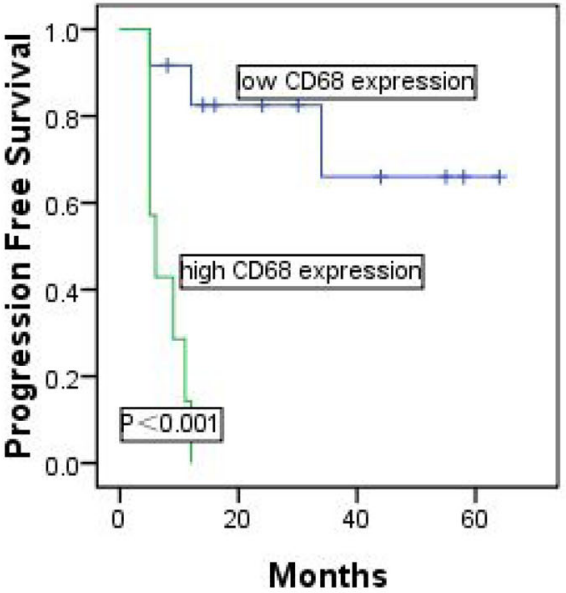

D

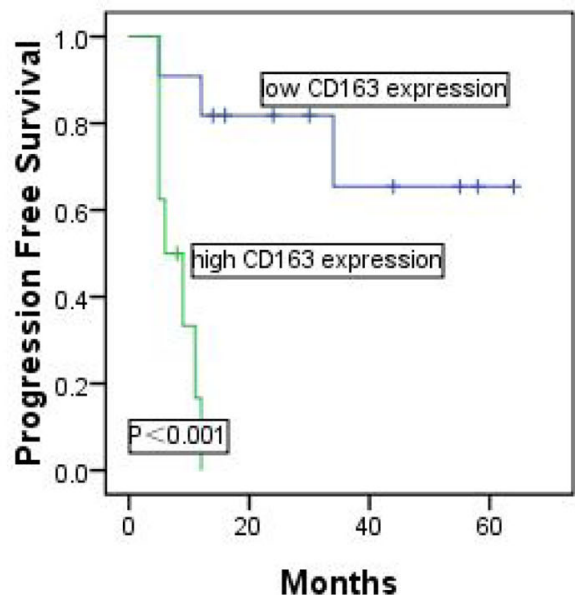

Fig. 5 Patients treated with R-CHOP identified by IPI score as IPI 2-3 were further stratified based on (a,b) CD68 expression and (c,d) CD163 expression, respectively

treatment given. In summary, TAMs may play an important role in promoting tumor growth, invasion and metastasis in TME of DLBCL, and CD163, as considered to be a marker relatively specific to M2 TAMs may serve as a better predictor than the pan-macrophage marker, CD68. There have been several studies on the role of TAMs in DLBCL. TAMs remodel extracellular matrix (ECM) remodeling and promote angiogenesis through producing matrix metalloprotein-9 (MMP-9), legumain, and vascular endothelial growth factor (VEGF) $[25,26]$. Reactive oxygen species (ROS) from TAMs contributes to DLBCL progression and drug resistance by increasing the expression of CD44 [27]. TAMs express programmed death-1 ligand (PD-L1), which induces the apoptosis of $\mathrm{T}$ cells, involving immunosuppression and immune evasion $[28,29]$. DLBCL cells can avoid phagocytosis through $\mathrm{CD} 47 /$ signal regulatory protein $\alpha$ (SIRP $\alpha)$ on TAMs pathway [30].
Monocytes and their progeny, promote tumor growth and angiogenesis, and involve in the suppression of host anti-tumor immunity. So more and more studies have found that elevation of peripheral monocytes is an adverse prognostic factor in many solid tumors [31-34], including DLBCL [14-20]. We have previously reported that peripheral AMC at diagnosis predicted outcome for DLBCL patients who received standard first-line regimens [14], elevation of AMC at the first relapse was an adverse prognostic factor for survive in relapsed/refractory DLBCL [15], and increased AMC tested during follow-up after standard first-line regimens was a risk factor for predicting recurrence of DLBCL [16]. Until now, few studies [34-37] have demonstrated that peripheral AMC significantly correlated with TAMs. However, to our knowledge, in DLBCL the correlation between AMC and TAMs has not been clarified. In our study, AMC was significantly positively correlated with 
TAMs in DLBCL patients. So, why peripheral blood monocytosis indicates poor prognosis in DLBCL, we deduce that monocytes differentiated into MPs after recruitment from peripheral blood to TME, then polarized into M2 phenotype thereby causing the progression of disease.

There are some limitations in the study. Firstly, as a retrospective study, patient selection might have been biased, and other unrecognized bias might have influenced the results. Secondly, the amount of cases is relatively small to prove conclusions. Thirdly, we used ROC curves and AUC to determine the best TAMs (CD68 + cells and CD163 + cells) cutoff value in our study, however, the cut-point for TAMs need investigation in other independent cohort.

\section{Conclusions}

In conclusion, we confirm the prognostic implications of TAMs in DLBCL in a retrospective study. Our results show that higher levels of TAMs in TME tend to have poorer outcomes, even in the rituximab era. Our findings, in conjunction with other previous studies, establish TAMs to be important in the DLBCL TME. As far as we know, this study is the first to identify the correlation between TAMs and AMC in DLBCL. Therapeutic strategies to inhibit M2 polarization may, therefore, be a potential target for anticancer therapy in DLBCL.

\section{Supplementary information}

Supplementary information accompanies this paper at https://doi.org/10. 1186/s12885-019-6208-x

Additional file 1: Figure S1. Receiver operating characteristic curve and area under the curve for (A) CD68+ cells/HPF, (B) CD163+ cells/HPF. Figure S2. CD68 and CD163 expression in DLBCL. (A) Frequencies of CD68 expression level in IPI score $=0-1,2-3$, and 4-5, (B) Frequencies of CD163 expression level in IPI score $=0-1,2-3$, and $4-5$.

\section{Abbreviations}

AMC: Absolute monocyte count; CHOP: Cyclophosphamide, hydroxydaunorubicin, vincristine, prednisone; Cl: Confidence interval; ECOGPS: Eastern Cooperative Oncology Group performance status; HR: Hazard ratio; IPI: International Prognostic Index; LDH: Lactate dehydrogenase; OS: Overall survival; PFS: Progression-free survival; R-CHOP: Rituximabcyclophosphamide, hydroxydaunorubicin, vincristine, prednisone

\section{Acknowledgments}

We thank all patients and their families for their participation in this study.

\section{Authors' contributions}

Y-LL designed the study, performed the statistical analysis, and drafted the manuscript. Z-HS, XW and K-SG participated in the collection of the clinical data. Z-MZ conceived of the study, and participated in its design and coordination and helped to draft the manuscript. All authors read and approved the final manuscript.

\section{Funding}

This study was supported by grants from National Natural Science Foundation of China (81700194), College Natural Science Foundation of Anhui Province (KJ2016A328) and Scientific Research of BSKY from Anhui
Medical University (XJ201511). These projects provide financial support for this experiment. In addition, it did not obtain other funding.

\section{Availability of data and materials}

The datasets used and/or analyzed during the current study are available from the corresponding author on reasonable request.

\section{Ethics approval and consent to participate}

This study was carried out in accordance with the principles of the Helsinki Declaration and approved by the ethics committee of the first affiliated and the second affiliated hospitals of Anhui Medical University. Each patient had signed informed consent.

\section{Consent for publication}

Not applicable.

\section{Competing interests}

The authors declare that they have no competing interests.

\section{Author details}

${ }^{1}$ Department of Pathology, Anhui Medical University, Hefei, Anhui 230032, People's Republic of China. ${ }^{2}$ Department of Pathology, The First Affiliated Hospital of Anhui Medical University, Hefei, Anhui 230022, People's Republic of China. ${ }^{3}$ Department of Pathology, Anhui Ji Min Cancer Hospital, Hefei, Anhui 230012, People's Republic of China. ${ }^{4}$ Department of Pathology, The Second Affiliated Hospital of Anhui Medical University, Hefei, Anhui 230601, People's Republic of China. ${ }^{5}$ Department of Oncology, The First Affiliated Hospital of Anhui Medical University, Hefei, Anhui 230022, People's Republic of China. ${ }^{6}$ Department of Hematology, The Second Affiliated Hospital of Anhui Medical University, Hefei, Anhui 230601, People's Republic of China.

Received: 23 March 2019 Accepted: 25 September 2019

Published online: 06 November 2019

\section{References}

1. Sarkozy C, Sehn LH. Management of relapsed/refractory DLBCL. Best Pract Res Clin Haematol. 2018:31(3):209-16.

2. Clappaert EJ, Murgaski A, Van Damme H, Kiss M, Laoui D. Diamonds in the rough: harnessing tumor-associated myeloid cells for cancer therapy. Front Immunol. 2018;9:2250.

3. Mantovani A, Marchesi F, Malesci A, Laghi L, Allavena P. Tumour associated macrophages as treatment targets in oncology. Nat Rev Clin Oncol. 2017; 14(7):399-416

4. $\quad$ Yang M, McKay D, Pollard JW, Lewis CE. Diverse functions of macrophages in different tumor microenvironments. Cancer Res. 2018;78(19):5492-503.

5. Hasselblom S, Hansson U, Sigurdardottir M, Nilsson-Ehle H, Ridell B, Andersson PO. Expression of CD68+ tumor-associated macrophages in patients with diffuse large B-cell lymphoma and its relation to prognosis. Pathol Int. 2008;58(8):529-32.

6. Cai QC, Liao H, Lin SX, Xia Y, Wang XX, Gao Y, et al. High expression of tumor-infiltrating macrophages correlates with poor prognosis in patients with diffuse large B-cell lymphoma. Med Oncol. 2012;29(4):2317-22.

7. Wada N, Zaki MA, Hori Y, Hashimoto K, Tsukaguchi M, Tatsumi Y, et al. Osaka lymphoma study group. Tumour-associated macrophages in diffuse large B-cell lymphoma: a study of the Osaka. Histopathology. 2012;60(2):313-9.

8. Nam SJ, Go H, Paik JH, Kim TM, Heo DS, Kim CW, et al. An increase of M2 macrophages predicts poor prognosis in patients with diffuse large B-cell lymphoma treated with rituximab, cyclophosphamide, doxorubicin, vincristine and prednisone. Leuk Lymphoma. 2014;55(11):2466-76.

9. Riihijärvi S, Fiskvik I, Taskinen M, Vajavaara H, Tikkala M, Yri O, et al. Prognostic influence of macrophages in patients with diffuse large B-cell Iymphoma: a correlative study from a Nordic phase II trial. Haematologica. 2015;100(2):238-45.

10. Marchesi F, Cirillo M, Bianchi A, Gately M, Olimpieri OM, Cerchiara E, et al. High density of CD68+/CD163+ tumour-associated macrophages (M2-TAM) at diagnosis is significantly correlated to unfavorable prognostic factors and to poor clinical outcomes in patients with diffuse large B-cell lymphoma. Hematol Oncol. 2015;33(2):110-2. 
11. Marinaccio C, Ingravallo G, Gaudio F, Perrone T, Nico B, Maoirano E, et al. Microvascular density, CD68 and tryptase expression in human diffuse large B-cell lymphoma. Leuk Res. 2014;38(11):1374-7.

12. Wang J, Gao K, Lei W, Dong L, Xuan Q, Feng M, et al. Lymphocyte-tomonocyte ratio is associated with prognosis of diffuse large B-cell lymphoma: correlation with CD163 positive M2 type tumor-associated macrophages, not PD-1 positive tumor-infiltrating lymphocytes. Oncotarget. 2017:8(3):5414-25.

13. Matsuki E, Bohn OL, El Jamal S, Pichardo JD, Zelenetz AD, Younes A, et al. Lymphocyte-to-monocyte ratio may serve as a better prognostic indicator than tumor-associated macrophages in DLBCL treated with rituximab. Appl Immunohistochem Mol Morphol. 2018. https://doi.org/10.1097/PAl. 0000000000000645.

14. Li YL, Pan YY, Jiao Y, Ning J, Fan YG, Zhai ZM. Peripheral blood lymphocyte/monocyte ratio predicts outcome for patients with diffuse large B cell lymphoma after standard first-line regimens. Ann Hematol. 2014;93(4):617-26.

15. Li YL, Gu KS, Pan YY, Jiao Y, Zhai ZM. Peripheral blood lymphocyte/ monocyte ratio at the time of first relapse predicts outcome for patients with relapsed or primary refractory diffuse large B-cell lymphoma. BMC Cancer. 2014;14:341.

16. Yan-Li L, Kang-Sheng G, Yue-Yin P, Yang J, Zhi-Min Z. The lower peripheral blood lymphocyte/monocyte ratio assessed during routine follow-up after standard first-line chemotherapy is a risk factor for predicting relapse in patients with diffuse large B-cell lymphoma. Leuk Res. 2014;38(3):323-8.

17. Watanabe R, Tomita N, Itabashi M, Ishibashi D, Yamamoto E, Koyama S, et al. Peripheral blood absolute lymphocyte/monocyte ratio as a useful prognostic factor in diffuse large B-cell lymphoma in the rituximab era. Eur J Haematol. 2014;92(3):204-10.

18. Nitta H, Terui $Y$, Yokoyama M, Mishima Y, Nishimura N, Ueda K, et al. Absolute peripheral monocyte count at diagnosis predicts central nervous system relapse in diffuse large B-cell lymphoma. Haematologica. 2015; 100(1):87-90.

19. Mo H, Shi Y, Han X, Zhou S, He X, Liu P, et al. Absolute monocyte count is a prognostic indicator in a patient with diffuse large B-cell lymphoma after autologous peripheral blood stem cell transplant. Leuk Lymphoma. 2015; 56(2):515-7.

20. Chen Y, Neelapu S, Feng L, Bi W, Yang TH, Wang M, et al. Prognostic significance of baseline peripheral absolute neutrophil, monocyte and serum $\beta 2$ - microglobulin level in patients with diffuse large b-cell lymphoma: a new prognostic model. Br J Haematol. 2016;175(2):290-9.

21. Swerdlow SH, Campo E, Harris NL, Jaffe ES, Pileri SA, Stein H, et al. World Health Organization Classification of Tumors of Haematopoietic and Lymphoid Tissues. 4th revised ed. Lyon: IARC; 2017.

22. A predictive model for aggressive NHL. The International non-Hodgkin's Lymphoma Prognostic Factors Project. N Engl J Med. 1993;329(14):987-94.

23. Quail DF, Joyce JA. Microenvironmental regulation of tumor progression and metastasis. Nat Med. 2013;19(11):1423-37.

24. Steidl C, Lee T, Shah SP, Farinha P, Han G, Nayar T, et al. Tumor-associated macrophages and survival in classic Hodgkin's lymphoma. New Engl J Med. 2010;362(10):875-85.

25. Shen L, Li H, Shi Y, Wang D, Gong J, Xun J, et al. M2 tumour-associated macrophages contribute to tumour progression via legumain remodelling the extracellular matrix in diffuse large B cell lymphoma. Sci Rep. 2016;6:30347.

26. Duletić-Načinović $A$, Gačić $V$, Valković $T$, Lučin $K$, Fišić E, Žuvić-Butorac M, et al. Concurrent elevations of VEGF, osteopontin and MCP-1 serum levels are independent predictors of survival in patients with diffuse large B-cell lymphoma. Acta Haematol. 2016;136(1):52-61.

27. Xu ZZ, Fu WB, Jin Z, Guo P, Wang WF, Li JM. Reactive oxygen species mediate oridonin-induced apoptosis through DNA damage response and activation of JNK pathway in diffuse large B cell lymphoma. Leuk Lymphoma. 2016;57(4):888-98.

28. Xu-Monette ZY, Xiao M, Au Q, Padmanabhan R, Xu B, Hoe N, et al. Immune profiling and quantitative analysis decipher the clinical role of immunecheckpoint expression in the tumor immune microenvironment of DLBCL. Cancer Immunol Res. 2019;7(4):644-57.

29. Vari F, Arpon D, Keane C, Hertzberg MS, Talaulikar D, Jain S, et al. Immune evasion via PD-1/PD-L1 on NK-cells and monocyte/macrophages is more prominent in Hodgkin lymphoma than DLBCL. Blood. 2018;131(16):1809-19.

30. Weiskopf K, Anderson KL, Ito D, Schnorr PJ, Tomiyasu H, Ring AM, et al. Eradication of canine diffuse large B-cell lymphoma in a murine xenograft model with CD47 blockade and anti-CD20. Cancer Immunol Res. 2016:4(12):1072-87.

31. Hu S, Zou Z, Li H, Zou G, Li Z, Xu J, et al. The preoperative peripheral blood monocyte count is associated with liver metastasis and overall survival in colorectal cancer patients. PLoS One. 2016;11(6):e0157486.

32. Shibutani M, Maeda K, Nagahara H, Iseki Y, Ikeya T, Hirakawa K. Prognostic significance of the preoperative lymphocyte-to-monocyte ratio in patients with colorectal cancer. Oncol Lett. 2017;13(2):1000-6.

33. Matsuo K, Hom MS, Moeini A, Machida H, Takeshima N, Roman LD, et al. Significance of monocyte counts on tumor characteristics and survival outcome of women with endometrial cancer. Gynecol Oncol. 2015;138(2):332-8

34. Kumagai S, Marumo S, Shoji T, Sakuramoto M, Hirai T, Nishimura T, et al. Prognostic impact of preoperative monocyte counts in patients with resected lung adenocarcinoma. Lung Cancer. 2014;85(3):457-64.

35. Shibutani M, Maeda K, Nagahara H, Fukuoka T, Nakao S, Matsutani S, et al. The peripheral monocyte count is associated with the density of tumorassociated macrophages in the tumor microenvironment of colorectal cancer: a retrospective study. BMC Cancer. 2017;17(1):404.

36. Li Z, Xu Z, Huang Y, Zhao R, Cui Y, Zhou Y, et al. The predictive value and the correlation of peripheral absolute monocyte count, tumor-associated macrophage and microvessel density in patients with colon cancer. Medicine (Baltimore). 2018;97(21):e10759.

37. Koh YW, Shin SJ, Park C, Yoon DH, Suh C, Huh J. Absolute monocyte count predicts overall survival in mantle cell lymphomas: correlation with tumour associated macrophages. Hematol Oncol. 2014;32(4):178-86.

\section{Publisher's Note}

Springer Nature remains neutral with regard to jurisdictional claims in published maps and institutional affiliations.

Ready to submit your research? Choose BMC and benefit from:

- fast, convenient online submission

- thorough peer review by experienced researchers in your field

- rapid publication on acceptance

- support for research data, including large and complex data types

- gold Open Access which fosters wider collaboration and increased citations

- maximum visibility for your research: over $100 \mathrm{M}$ website views per year

At BMC, research is always in progress.

Learn more biomedcentral.com/submissions 